\title{
Human Vasculogenesis Ex Vivo: Embryonal Aorta as a Tool for Isolation of Endothelial Cell Progenitors
}

\author{
Giulio Alessandri, Marina Girelli, Gianluca Taccagni, Augusto Colombo, \\ Roberto Nicosia, Arnaldo Caruso, Manuela Baronio, Stefano Pagano, Lidia Cova, \\ and Eugenio Parati
}

\begin{abstract}
Laboratory of Microbiology (GA, AC, MB), University of Brescia, Brescia; Centro Scompenso Cardiaco (GA), Civic Hospital, Brescia, Italy; Department of Pathology (GT), San Raffaele Hospital and Scientific Institute, Milan; Institute for Obstetrics and Gynaecology "L Mangiagalli" (AC), Milan, Italy; Division of Pathology and Laboratory Medicine $(R N)$, Veterans Affairs Puget Sound Health Care System and Department of Pathology (RN), University of Washington, Seattle, Washington; and Neuropharmacology (MG, SP, LC, EP), National Neurological Institute "C. Besta," Milan, Italy
\end{abstract}

\begin{abstract}
SUMMARY: Vasculogenesis, the de novo formation of new blood vessels from undifferentiated precursor cells or angioblasts, has been studied with experimental in vivo and ex vivo animal models, but its mechanism is poorly understood, particularly in humans. We used the aortic ring assay to investigate the angioforming capacity of aortic explants from 11- to 12-week-old human embryos. After being embedded in collagen gels, the aorta rings produced branching capillary-like structures formed by mesenchymal spindle cells that lined a capillary-like lumen and expressed markers of endothelial differentiation (CD31, CD34, von Willebrand factor [VWF], and fms-like tyrosine kinase-1 [Flk-1]/vascular endothelial growth factor receptor 2 [VEGFR2]). The cell linings of these structures showed ultrastructural evidence of endothelial differentiation. The neovascular proliferation occurred primarily in the outer aspects of aortic rings, thus suggesting that the new vessels mainly arose from immature endothelial precursor cells localized in the outer layer of the aortic stroma, ie, a process of vasculogenesis rather than angiogenesis. The undifferentiated mesenchymal cells (CD34+/CD31-), isolated and cultured on collagen-fibronectin, differentiated into endothelial cells expressing CD31 and vWF. Furthermore, the CD34+/CD31+ cells were capable of forming a network of capillary-like structures when cultured on Matrigel. This is the first reported study showing the ex vivo formation of human microvessels by vasculogenesis. Our findings indicate that the human embryonic aorta is a rich source of CD34+/CD31- endothelial progenitor cells (angioblasts), and this information may prove valuable in studies of vascular regeneration and tissue bioengineering. (Lab Invest 2001, 81:875-885).
\end{abstract}

\begin{abstract}
A ngiogenesis (the sprouting of new blood vessels from the differentiated endothelium of preexisting vessels) plays an important role in physiologic and pathologic processes (Folkman, 1985). Angiogenesis occurs normally during embryonic development, tissue regeneration, and the menstrual cycle and pathologically in cancer, proliferative retinopathy, and rheumatoid arthritis (Folkman and Shing, 1992). In contrast, vasculogenesis (the de novo formation of new blood vessels from undifferentiated precursor cells or angioblasts) occurs mainly during embryonic development (Risau, 1995). Vasculogenesis has been studied with experimental in vivo and ex vivo animal models, but its mechanisms, particularly in humans, are poorly understood (LeDourain, 1973; Pardanaud et
\end{abstract}

Received February 23, 2001

GA and MG contributed equally to this work. EP is supported by Ministry of Health, Italy, Grant 030.3/FR99.39. RN is supported by National Institutes of Health (NIH) Grant HL52585.

Address reprint requests to: Dr. Eugenio Parati, Istituto Nazionale Neurologico C. Besta, Via Celoria, 11, 20133 Milan, Italy. E-mail: parati@istituto-besta.it al, 1987; Risau and Flamme, 1995). In an attempt to fill this gap, we used the aortic ring assay developed by Nicosia (Nicosia and Ottinetti, 1990; Nicosia et al, 1982) to investigate the angioforming capacity of aortic explants from human embryos and the role of vasculogenesis in this process.

\section{Results}

\section{Outgrowth of Vascular-Like Cords from Aorta Ring Explants}

Aortic ring explants from 11- to 12-day-old embryos embedded in collagen gels (Elsdale and Bard, 1972; Nicosia and Ottinetti, 1990) were observed for up to 10 days. During the first 24 hours, there were no significant changes, although a few spindly fibroblast-like cells migrated into the matrix. Over the subsequent 24 hours, cohesive cellular cords began to sprout from the aortic rings (Fig. 1, a, b, and c). The number of cord-like structures was highly variable and difficult to quantify because of the complexity of the threedimensional outgrowth and abundant fibroblast-like cells. Few cords appeared to arise from the cut edges 

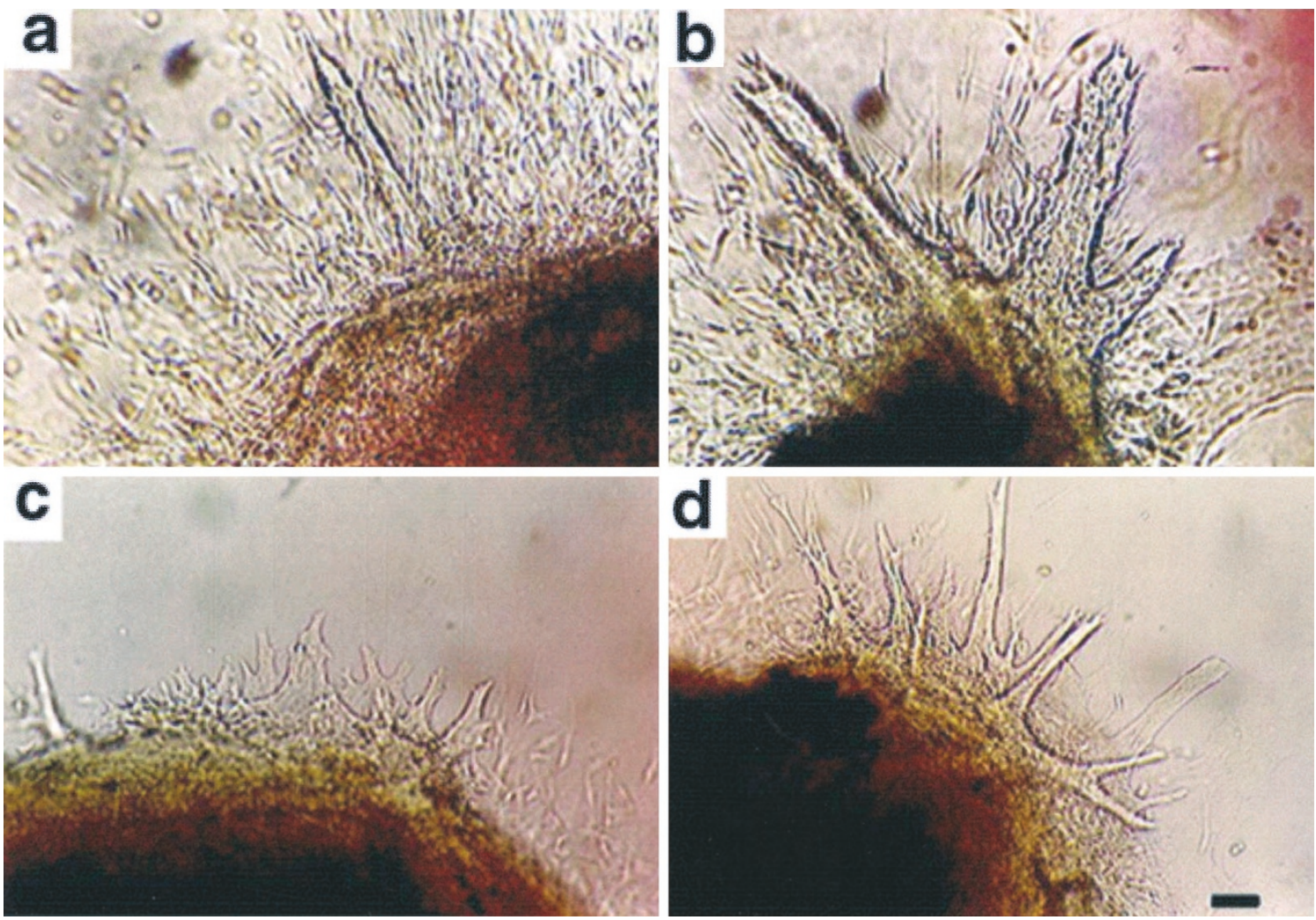

Figure 1.

Micrographs of collagen gel cultures of various human embryonic aorta explants. Vascular outgrowths arising from them are shown at different times of culture. a, A single capillary-like structure surrounded by spindly mesenchymal cells after 48 hours of culture. b. Three large cords arising from the same area of an aortic explant, photographed after 48 hours of culture. c, Incipient buds emerging from a wide area of an aortic explant cultured in collagen for 48 hours. $d$, Outgrowths of branching capillary-like tubes after 72 hours of culture. The cords grow haphazardly and divide into branches to form a more complex arborizing pattern than at the earlier stages seen in Panels a, b, and c. Scale bars: $25 \mu \mathrm{m}(\mathrm{a}, \mathrm{b}), 50 \mu \mathrm{m}(\mathrm{c}, \mathrm{d})$.

of the explant, whereas most emerged from adventitial or intimal surfaces. By the third day, the cords had grown haphazardly, dividing into branches and forming complex arborizing patterns (Fig. 1d). At this time some of the newly formed cords had regressed, whereas others were still forming and developing into capillary-like structures. Maximum cord elongation (2-3 $\mathrm{mm}$ ) was observed after five days. This was followed by rapid regression, which was complete toward the end of the week. These findings were recorded in $70 \%$ of the cultures $(n=15)$.

\section{Characterization of Embryonic Aorta}

Histological and immunohistochemical analyses were conducted to characterize embryonic aortic tissue before the in vitro culture in collagen gel. The embryonic aorta wall consisted of a well-formed endothelial lining, an internal elastic lamina, and several external layers of spindly mesenchymal cells. These cells were arranged in compact fascicles and had ultrastructural features of poorly differentiated smooth muscle, including small bundles of myofilaments and surrounding elastic fibers (Fig. 2). At this time of embryonic development, vasa vasorum were absent (Gilbert, 1997).

Immunohistochemistry showed that only the cells lining the lumen stained strongly for CD31(Fig. 2a) and von Willebrand factor (vWF) (data not shown), thus confirming their mature endothelial phenotype. None of the other cells constituting the aortic wall presented a specific immunoreactivity for these antibodies. The staining with CD34, an antigen typically expressed in endothelial progenitor cells (Flamme and Risau, 1992; Weiss and Orkin, 1996), clearly showed that not only the lumen, but also the more peripheral/para-aortic cells, expressed this antigen. In a serial section of the same explant used for CD31 analysis, CD34 seems to be expressed by the para-aortic tissue (Fig. 2b), whereas, in several sections of different rings, it seems to be constitutively and specifically expressed by the external cells of the aortic wall (Fig. 2c), suggesting that it is not, or not only, a para-aortic cell feature. The external cell layer furthermore showed a strong reactivity with antibody for fms-like tyrosine kinase-1 (Flk1)/vascular endothelial growth factor receptor 2 (VEGFR2), another marker of endothelial immaturity (Hirashima et al, 1999; Yamashita et al, 2000), which was also expressed by endothelial cells lining the lumen (Fig. 2d).

\section{Characterization of Vascular-Like Cords}

The cellular phenotype of cords and capillary tubes arising from aortic rings was investigated by light microscopy, immunohistochemistry, and electron mi- 

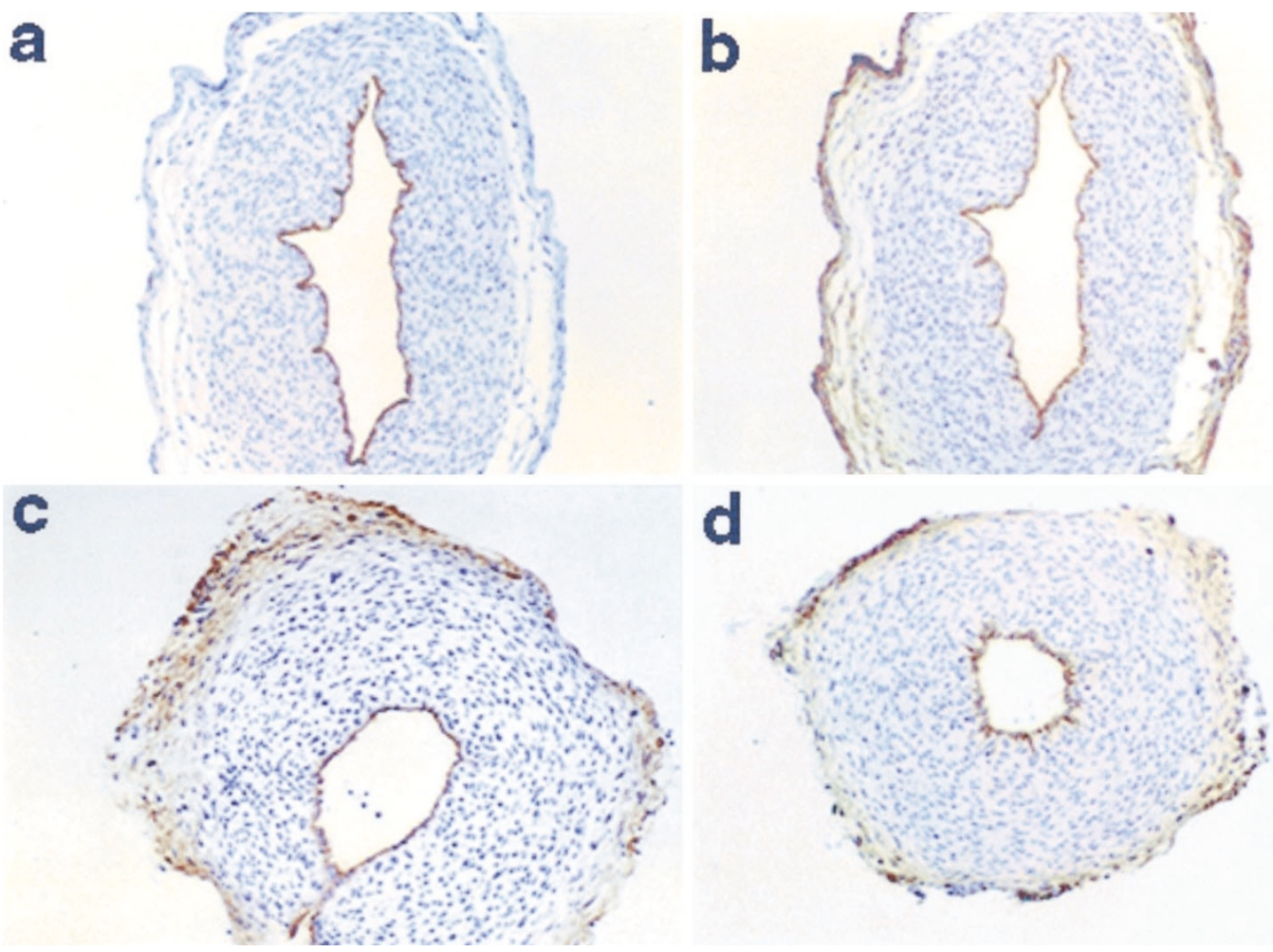

Figure 2.

Immunohistochemical characterization of untreated human embryonic aortae. The tissues were immediately fixed after recovery and then embedded in paraffin and cross-sectioned. a, Aortic cross-section stained for CD31 antigen, showing that only the endothelial cells lining the lumen presented a strong and clear immunoreactivity for this antibody. The other cells constituting the aortic wall were totally negative. b and c, Two representative sections of different explants immunostained for CD34. b, Serial cross-section of the same aortic explant shown in Panel a. Not only did the endothelial cells lining the lumen display an intense positive staining for CD34 antibody, but so also did the more peripheral cell layer that, in some sections, seemed to be a para-aortic tissue (b), in some others, a constitutive layer of cells in the aortic external wall (c). $d$, The same layers of cells that positively stained for CD34 in Panels b and c also expressed fms-like tyrosine kinase-1 (Flk-1), as shown by the immunohistochemistry conducted on a different tissue section. Scale bar: $160 \mu \mathrm{m}$.

croscopy. The outgrowths consisted of mesenchymal spindle cells, sometimes forming aggregates with central necrotic cores (Fig. 3a). Incipient formation of capillary-like structures was often evident in areas where mesenchymal cells were densely packed (Fig. $3, \mathrm{~b}$ and $\mathrm{c}$ ). Cohesive cells with abundant cytoplasm and prominent nuclei lined the capillary-type lumen of these outgrowths. These endothelial-like cells tended to form delicate networks of long straight channels that sometimes branched at an acute angle (Fig. 4a).

Immunochemistry showed that the cells lining the channels stained strongly for CD31 (Fig. 4, a and b) and CD34 (Fig. 4c). They were also immunoreactive for vWF, though less strongly (Fig. 4d). The surrounding mesenchymal cells, which were not organized in vascular structures, were consistently negative for all these markers.

Moreover, the cells forming neovessels, as well as the endothelial cells lining the lumen, stained strongly for Flk-1/VEGFR-2, as demonstrated by different magnification in Figure 4, e and f. Interestingly, Figure 4e shows one of the rare cases in which the microvessels seem to originate both from the internal endothelial layer and the periphery of the aortic section. This phenomenon was recorded in approximately 1 of 20 sections analyzed. In an elevated number of histological sections, in fact, the most of neovascular prolifer- ation occurred primarily in the outer aspects of the aortic rings (Fig. 4a), whereas, in a very limited number of cases, it seemed to originate in the endothelial lining of the aortic lumen (Fig. 4e).

These observations, together with the characteristics of the untreated embryonic aorta (Fig. 2), therefore suggest that neovessels arose mainly from immature endothelial precursor cells (CD34+/Flk1+) of the aortic external mesenchyme, ie, that vasculogenesis rather than angiogenesis was the main process responsible for the vascular outgrowth in these cultures.

By electron microscopy, the aortic outgrowth was found to be composed of an admixture of primitive mesenchymal cells (Fig. 5, a and b), endothelial-lined neovessels (Fig. 5, c and d), and cells with a mixed mesenchymal/endothelial phenotype suggesting differentiation of the mesenchyme into endothelium (Fig. $5 b)$. The lining of the neovessels was composed of differentiated endothelial cells connected by junctional complexes. Endothelial cells exhibited a welldefined luminal/abluminal polarity and rested on a thin and discontinuous basal lamina. The endothelial cytoplasm contained abundant rough endoplasmic reticulum (RER) with focally dilated cisternae, Golgi complexes, pinocytotic vesicles, mitochondria, free ribosomes, and secondary lysosomes including osmiophilic myelin figures (Fig. 5, c and d). Immature 


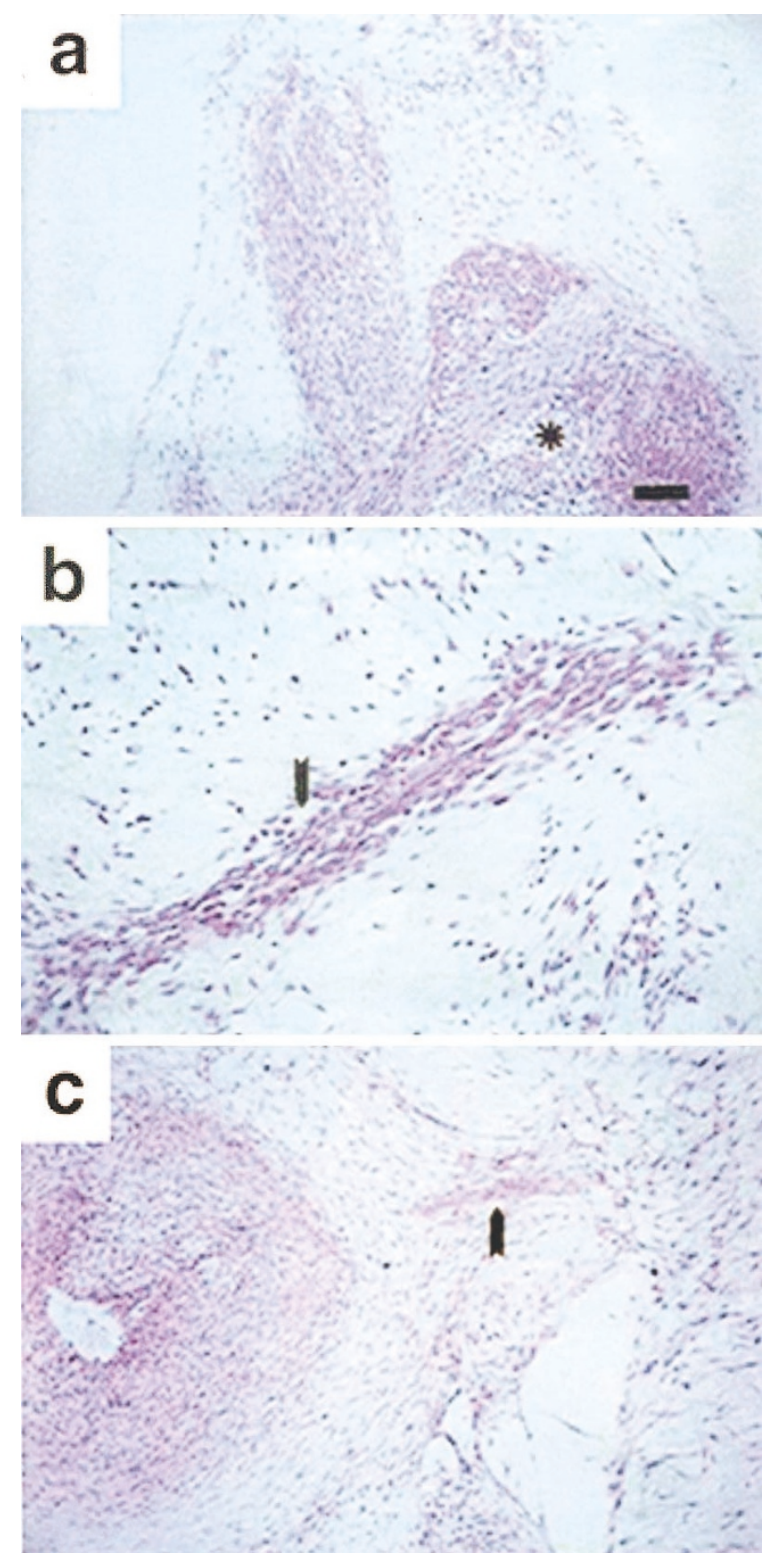

Figure 3.

Light microscopy of capillary-like structures sprouting from an aortic ring. a, Transverse section through an irregular bundle of densely packed mesenchymal cells with central necrotic area $\left(^{*}\right)$. b, Longitudinal section of digitiform mesenchymal outgrowth showing central mass of cells at an early stage of differentiation into capillary-like structures (arrow). c, Loosely organized proliferation of mesenchymal cells with developing capillary-like structure (arrow). This large cord may be an immature vessel structure and studies using a time-lapse recorder are in progress to better evaluate the origin. Scale bars: $100 \mu \mathrm{m}$ (a, c), $50 \mu \mathrm{m}$ (b).

mesenchymal cells contained abundant glycogen, mitochondria, and bundles of microfilaments with fusiform densities, which were particularly noticeable in subplasmalemmal locations (Fig. 5, a and b). Cells with a transitional phenotype between mesenchymal cells and endothelium tended to align in longitudinal arrays and establish junctional connections with one another. This caused the separation of newly formed luminal spaces from the surrounding extracellular matrix (Fig. 5b). Cells sequestered within vascular lumina as a result of these morphogenetic changes lost their anchorages to the surrounding matrix and died, leaving behind cytoplasmic debris that was eventually found within differentiated neovessels (Fig. 5c).

\section{Isolation of CD34+ Precursor-Endothelial Cells}

To isolate immature endothelial cells (CD34+Flk1+/ CD31-) present in the aortic wall, freshly dissected aortas were digested with collagenase-dispase solution. The resulting cell suspension was incubated first with CD31 antibody-coated magnetic beads to remove differentiated endothelial cells and then with beads coated with an antibody against CD34. This procedure demonstrated that less than $1 \%$ of cells were CD31-positive, whereas $29.5 \pm 2.5 \%$ of the cells were CD34-positive (average $1.2 \times 10^{6}$ cells isolated per aorta, $n=4)$. This result was confirmed by flow cytometry analysis (FACS) (Fig. 6).

To better characterize these cells at the time of their isolation, some of them were plated onto dishes coated with collagen-fibronectin (Asahara et al, 1997) and cultured for a short time (24 hours) to avoid differentiation in endothelial basal medium (EBM) supplemented with 10\% FCS and endothelial cell-growth supplement (Alessandri et al, 1998). The day after plating, these cells were fixed and stained with CD31 and vWF antibodies not showing immunoreactivity (Fig. 7a).

Furthermore, to determine whether selected CD34+/CD31 - cells could differentiate into mature endothelium, they were seeded and cultured for 7 to 10 days in the same conditions described above. The cells were then detached with trypsin and incubated with anti-CD31-coated beads. As a result of this treatment, $25 \pm 6.5 \%$ (mean of five experiments) of the initial CD34+/CD31- cell population differentiated into CD34+/CD31+ cells (Table 1) which also stained for CD31 and VWF by immunofluorescence (Fig. 7, b and c), thus demonstrating the formation of a more mature endothelial phenotype. Under the same culture conditions, none of the CD31-/CD34- cells differentiated into CD34+/CD31+ cells, nor presented the acquisition of specific immunoreactivity for VWF (data not shown). To further confirm the process of maturation, the CD34+ cells were cultured for 2 to 3 weeks and then seeded on Matrigel to evaluate their capability to form capillary-like tubular structures. In addition to the mature endothelial cells (CD31+) isolated from the aortic lumen and cultured under the same conditions, the CD34+/CD31+ cells were able to form a net of capillary-like structures after 24 hours of incubation (Fig. 7d)

\section{Discussion}

Despite the recent substantial increase in our understanding of the molecular mechanisms regulating embryonic vasculogenesis and angiogenesis, there is still much that needs to be learned, particularly in relation to humans. In an attempt to fill a part of this gap, we used the aortic ring assay (Nicosia and Ottinetti, 1990) 


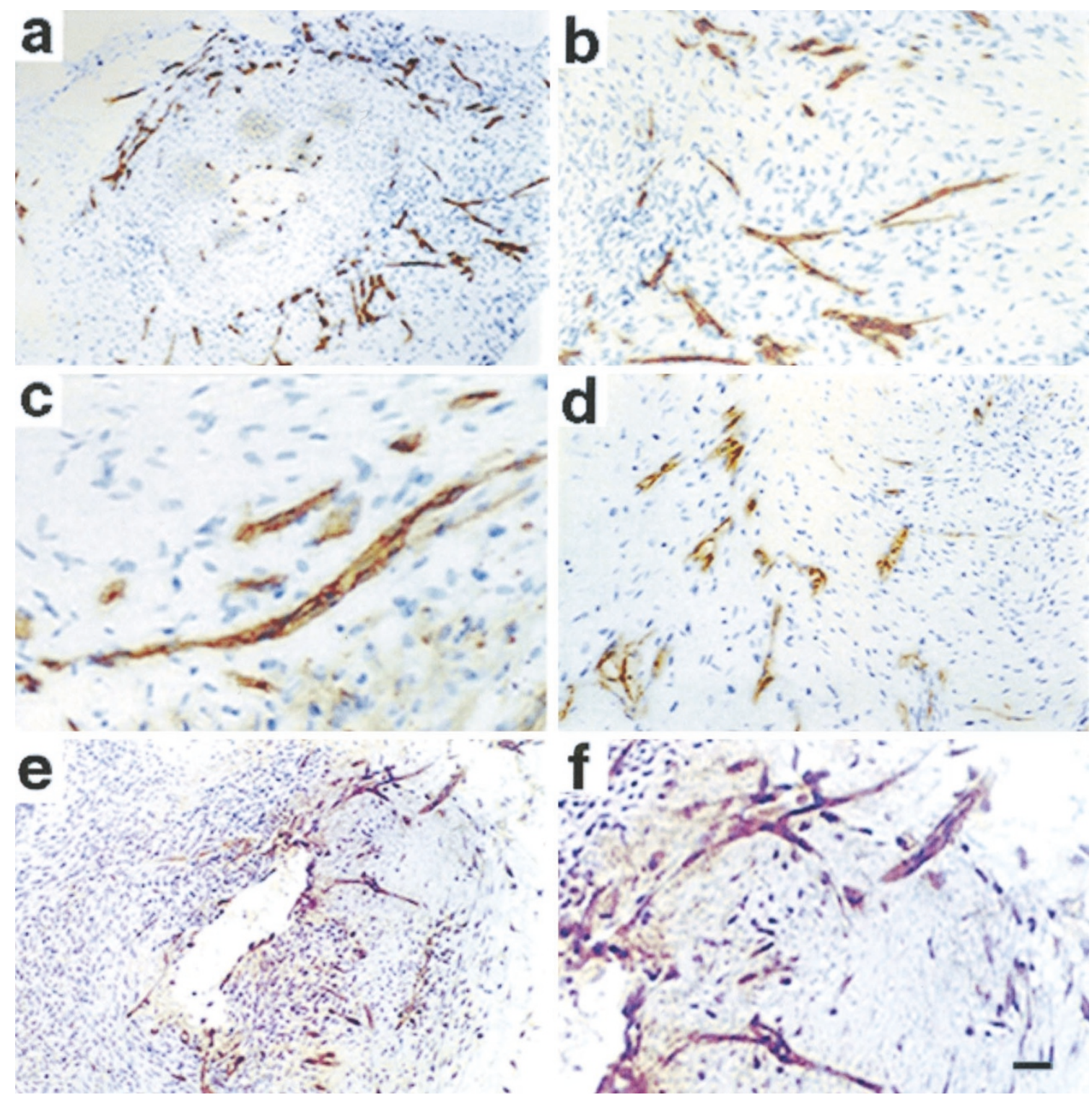

\section{Figure 4.}

Immunohistochemistry of capillary-like structures arising from different aortic rings paraffin-embedded after 72 to 96 hours of culture in collagen matrix. a, Cross-section of an aortic ring showing many CD31-positive cells organized into capillary-like channels. These outgrowths appear to arise from a mass of undifferentiated mesenchymal cells at the periphery of the explant. b. Higher magnification of a selected area of the same explant represented in Panel a showing the formation of long and branched channels. c, Capillary-like structures photographed at high magnification showing intense CD34 positivity; surrounding mesenchymal cells are negative. d, Cells of incipient capillary-like channels within a mass of undifferentiated mesenchymal cells are immunoreactive for von Willebrand factor (VWF). e and f, Cross-section of an aortic ring photographed at different magnification showing Flk-1 expression by endothelial cells lining the lumen and by cells organized to form vascular-like channels. In this rare case, the vascular structures seem to originate from the internal layer of endothelial cells. Scale bars: $100 \mu \mathrm{m}(\mathrm{a}, \mathrm{e}), 50 \mu \mathrm{m}(\mathrm{b}, \mathrm{d}, \mathrm{f}), 25 \mu \mathrm{m}(\mathrm{c})$.

to analyze the vasculogenesis properties of 11- to 12-week-old human embryonic aortae.

In brief, the results of this study showed that human embryonic aortic explants produce an intense outgrowth of capillary structures when cultured in a three-dimensional collagen matrix, the in vitro formation of which resembles the in vivo physiologic process of embryonic vasculogenesis. Our immunohistochemical and ultrastructural findings suggest that these outgrowths mainly arise as a result of the differentiation of immature CD34+ cells and not from pre-existing endothelial cells $(\mathrm{CD} 31+)$, although this latter process cannot be entirely excluded.
In fact, as shown by immunohistological data, immature CD34+ cells also express Flk-1/VEGFR-2, another marker highly expressed on the endothelial progenitors cells (Asahara et al, 1999; Hirashima et al, 1999; Peichev et al, 2000). They are spatially localized in the more external mesenchymal layer of cells constituting the aortic wall, in strict correlation with the para-aortic tissue, which however does not contain mature endothelial cells at this stage of development, as demonstrated by their negative staining for CD31.

To the best of our knowledge, this is the first report describing the ex vivo formation of human microvessels by vasculogenesis. A previous study conducted 

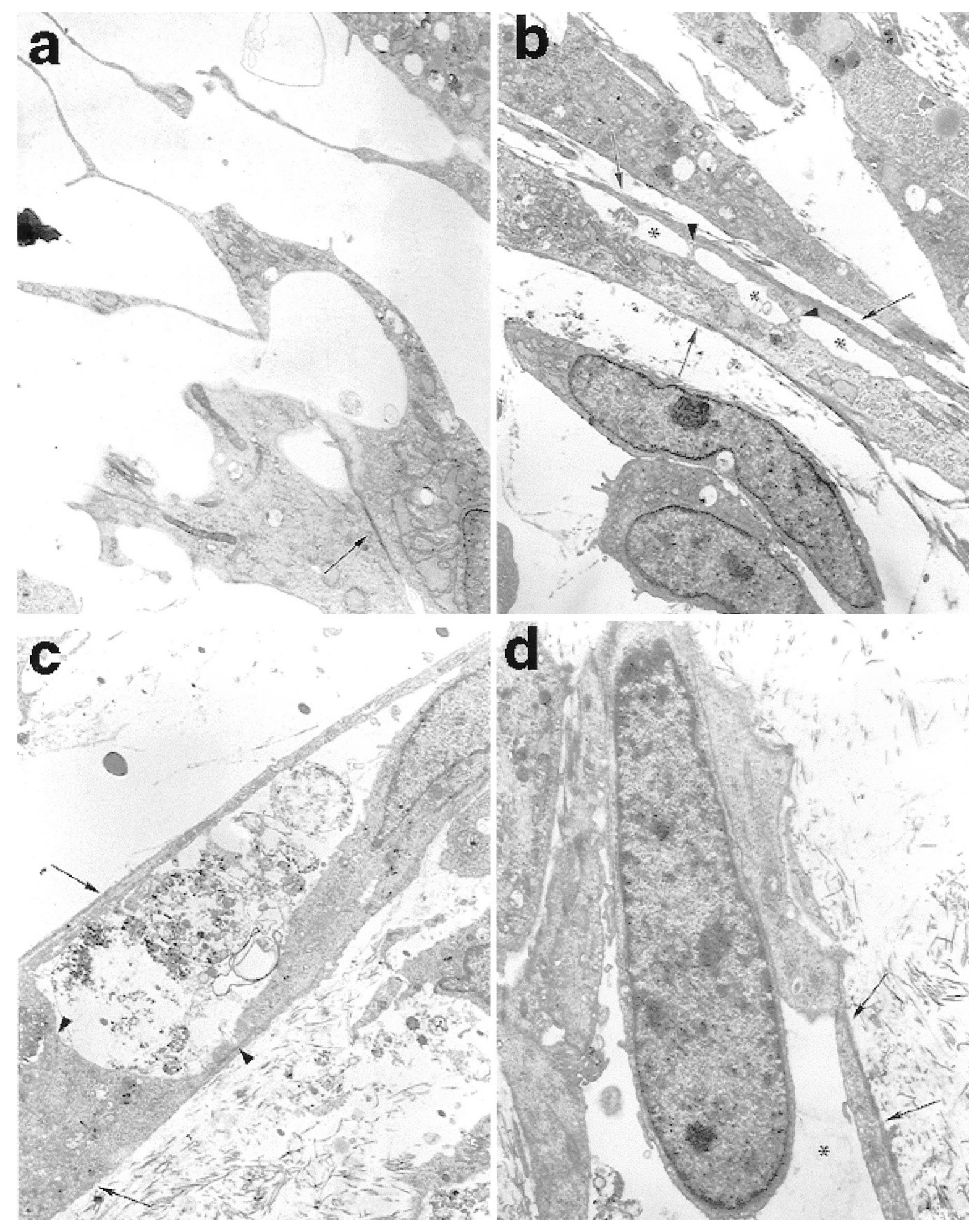

\section{Figure 5.}

Electron micrographs of cells from human embryonic aortic ring outgrowths. a, Primitive mesenchymal cells with dendritic cytoplasmic processes and occasional junctional complexes (arrow) $(\times 3000)$. b, Mesenchymal outgrowths showing focal longitudinal alignment of mesenchymal cells into cord-like structure (arrows). The cells, connected by junctional complexes (arrowheads), have surrounded a space free of extracellular matrix, which may represent a primitive lumen (asterisks) $(\times 3000)$. c, Neovessel lined by endothelial cells connected by junctional complexes (arrowheads). The lumen contains cell debris. Endothelial cells rest on a thin and discontinuous basal lamina (arrows) $(\times 3000)$. d, Neovessel surrounded by discontinuous basal lamina (arrow) and collagen fibrils. The endothelium exhibits prominent pinocytotic activity, junctional complexes, and well-defined luminal/abluminal polarity. The luminal space is marked by an asterisk ( $\times 3000$ ).

by other authors on fragments of fetal human placenta vessels did not investigate this origin of new capillary formation (Brown et al, 1996).
Our data also indicate that the process of endothelial cell (EC) differentiation in humans is different from that using mouse embryonic stem cells (Balconi et al, 


\section{Human embryonic aorta cell population: cytofluorimetryc analysis for CD31 and CD34 antigen expression}
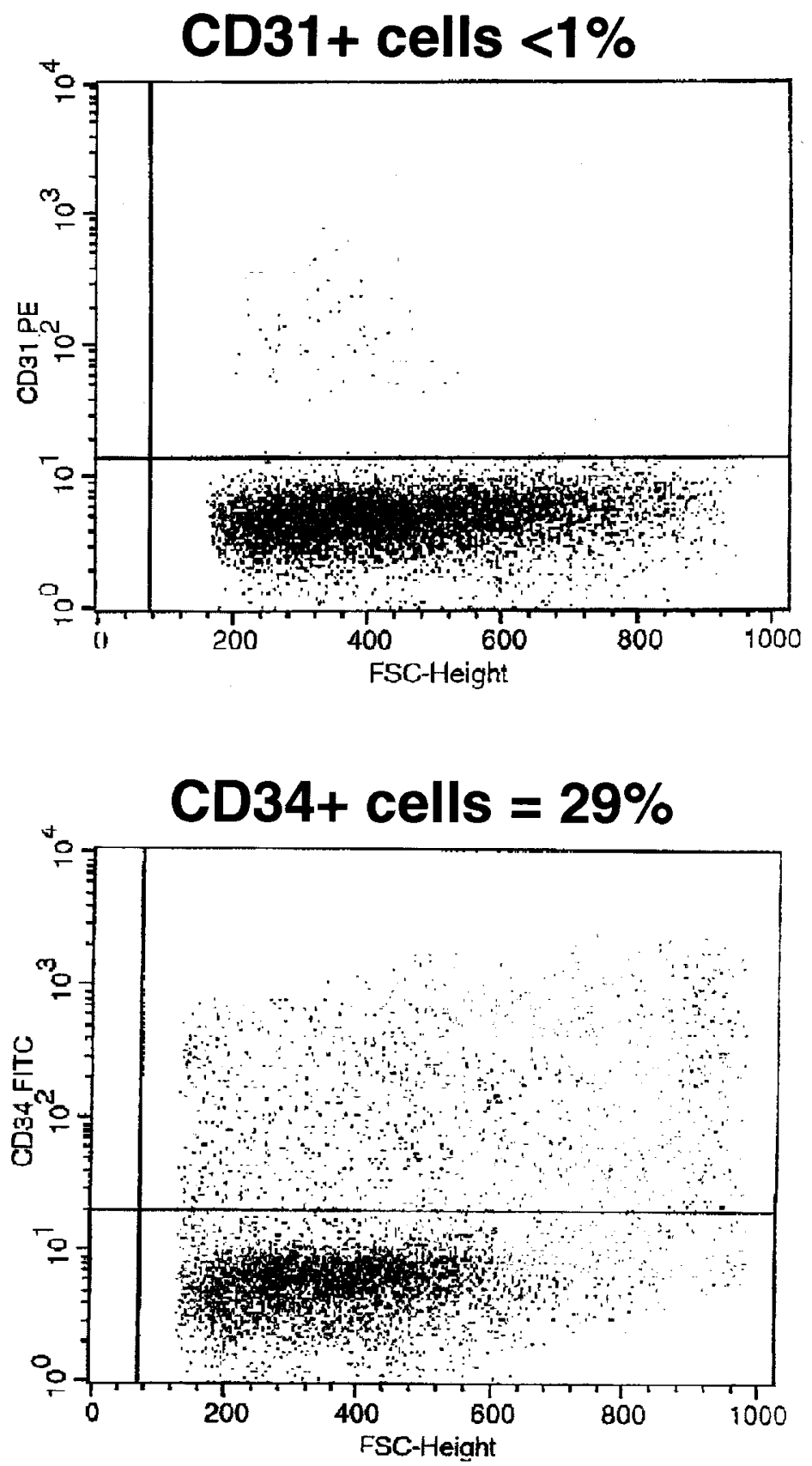

Figure 6.

Binding of anti-CD31 and anti-CD34 antibodies on total cell population obtained by enzymatic digestion of an aortic fragment, as measured by flow cytometry analysis (FACS). Binding of anti-CD31 antibody (upper panel) revealed that a small percentage of cells, approximately $1 \%$, express this antigen, according to the fact that only the cells lining the lumen present a mature endothelial phenotype. The same aortic cell suspension stained with CD34 antibody (lower panel) shows a higher percentage of immunoreactive cells (about $30 \%$ ). 

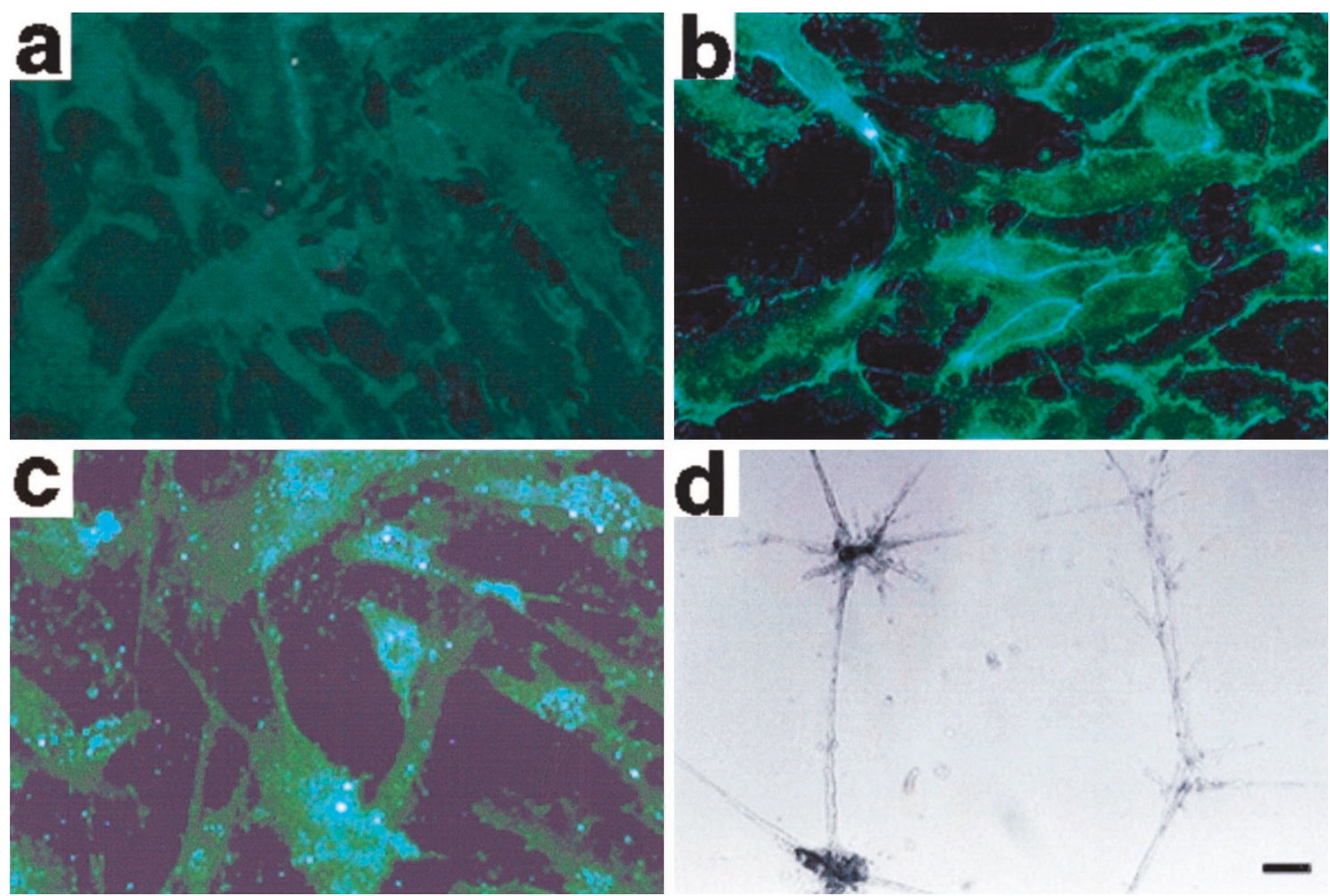

\section{Figure 7.}

Immunofluorescence staining of CD34+ cells and formation of capillary structures on Matrigel. Representative culture of CD34+ cells after 10 days of growth on collagen-fibronectin-coated chamber slide stained with anti-CD31(b) and anti-vWF (c). a, CD31 staining performed on CD34+ cells 24 hours after plating revealed no signal expression. At the same time in culture, vWF antigens were also absent. b, Light CD31 expression along the cell-cell contact borders. At the time of culture, usually $20 \%$ to $30 \%$ of CD34+ cells stained positively for CD31. c, Typical granular perinuclear positivity for vWF staining on CD34+ cells. Its signal intensity and distribution, but not its expression, are comparable to mature endothelial cells isolated from the adult human aorta (data not shown), confirming the immature endothelial phenotype of CD34+ cells. d, Capillary structures formed by CD34+/CD31+ cells 24 hours after seeding on Matrigel. The CD34+/CD31+ cells form tube-like structures, with long vessel-like extensions interconnecting occasional clumps of cells. Scale bars: $17 \mu \mathrm{m}(\mathrm{a}, \mathrm{b}, \mathrm{c}), 250 \mu \mathrm{m}$ (d).

Table 1. Differentiation of CD34+CD31- Cells into CD34+CD31+ Cells after 10 Days of Culture

\begin{tabular}{ccccc}
\hline \multicolumn{2}{c}{ DAY 0 } & & \multicolumn{2}{c}{ DAY 10} \\
\cline { 4 - 5 } $\begin{array}{c}\text { Antigen } \\
\text { selections }\end{array}$ & $\begin{array}{c}\text { \% of positive } \\
\text { cells }\end{array}$ & & $\begin{array}{c}\text { Antigen } \\
\text { expressions }\end{array}$ & $\begin{array}{c}\text { \% of positive } \\
\text { cells }\end{array}$ \\
\hline CD34+CD31+ & $1.3 \pm 0.5$ & & CD34+CD31+ & $25 \pm \mathbf{6 . 5}$ \\
CD34+CD31- & $29.5 \pm 2.5$ & & & \\
CD34-CD31- & $60.5 \pm 7$ & & CD34+CD31- & $69 \pm 8.3$ \\
\hline
\end{tabular}

2000; Yamashita et al, 2000). Indeed, in contrast to mouse stem cells, the acquisition of CD31 antigen in human vasculogenesis occurs after the maturation of CD34+ cells in culture. Furthermore, our findings indicate that the human embryonic aorta is a rich source of CD34+/CD31 - endothelial progenitor cells (angioblasts), which are localized all along the external layer of the aorta mesenchyme, probably in close contact with the para-aortic tissue, which at this stage of development does not contain mature endothelium. We found that only $20 \%$ to $30 \%$ of the CD34+ cells isolated from aorta give rise to more mature endothelium. The rest of CD34+ cells have not yet been investigated. A recent report of an investigation using mouse embryonic stem cells (Yamashita et al, 2000) suggests that endothelial and mural cells (pericytes and vascular smooth muscle) may originate from the same Flk-1+ precursors, but we do not know if this process occurs also in humans. However, we suggest that among embryonic aorta, it may be possible to find and isolate primitive CD34+ Flk-1+ cells that may differentiate into various vascular cell phenotypes. This may be an important new finding because it could lead to the isolation of vascular stem cells, and a number of previously published reports (reviewed by Fuchs and Segre, 2000) have indicated the enormous potential of multipotent stem cells in clinical applications.

Given the strict correlation between the formation and organization of the hematopoietic/endothelial (Nishikawa et al, 1998) and nervous systems, the possibility of combining the transplantation of angio- 
blasts/vascular stem cells and neural stem cells in neurodegenerative diseases represents an appealing approach toward improving the success of this innovative therapy. Furthermore, angioblasts/vascular stem cells could be used to investigate the molecular mechanism involved in human endothelial cell maturation and vasculogenesis, and our model may therefore also be valuable for vascular regeneration studies. Finally, these cells offer an important alternative for the clinical treatment of ischemia and other vascular diseases and suggest possibilities for tissue bioengineering (Niklason, 1999) and gene therapy (Mulligan, 1993).

\section{Materials and Methods}

\section{Preparation of Vascular Explants}

Permission to use human material was obtained from the ethical committees of the Neurological Institute " $\mathrm{C}$. Besta" and the Institute for Obstetrics and Gynaecology "L. Mangiagalli," Milan, Italy. The tissue was obtained following the ethical guidelines of the Network for European CNS Transplantation and Restoration (NECTAR), which is available online at www.nesu.mphy.lu.se/nectar.

Aortas from normal embryos that were legally aborted were obtained at the Obstetrics and Gynecology Department of L. Mangiagalli Hospital, Milan, Italy. The embryonic aortas were washed several times with PBS and cleaned, care being taken not to damage the vessel walls. Under a dissecting microscope, 1-mmthick rings were prepared and stored in DMEM at $4^{\circ} \mathrm{C}$ for not more than 2 hours before use.

The explants were placed in collagen gel using a published procedure with minor modifications (Nicosia and Ottinetti,1990). Briefly, 7 vol of type I collagen solution $(4 \mathrm{mg} / \mathrm{ml})$ prepared from rat tail (Elsdale and Bard, 1972), on ice, was mixed with 2 vol of endothelial basal medium (EBM) (BioWhittaker, Walkersville, Maryland), $5 \times$ normal strength, and 1 vol of HEPES (0.2 M). Each well of a 12 multiwell plate was filled with $0.7 \mathrm{ml}$ of collagen solution and left to gel in a humidified incubator at $37^{\circ} \mathrm{C}$ for 1 hour. One ring per well was placed on top of the gel and a further $0.5 \mathrm{ml}$ of collagen solution added to cover the material. After the second collagen layer had gelled, 1 to 2 hours later, $1 \mathrm{ml}$ of EBM growth medium (Alessandri et al, 1998) was added. The plates were incubated for up to 10 days. The medium was changed every 2 days.

\section{Isolation of Endothelial CD34+/CD31- Cells}

After removal, a section of aorta was rinsed several times with PBS, minced rapidly with scissors, and incubated overnight with $0.25 \%(\mathrm{w} / \mathrm{v})$ collagenasedispase solution (Boehringer, Mannheim, Germany). After centrifugation, approximately $1 \times 10^{5}$ cells were used for FACS analysis (CD31 and CD34 expression), and the remaining cells were incubated with magnetic beads coated with antibody to CD31 (DAKO, Carpinteria, California) (cell:bead ratio, 1:1). The CD31negative cells were recovered by centrifugation and further incubated with magnetic beads coated with antibody to CD34 (Dynal, Oslo, Norway). The CD34+/ CD31 - cells were recovered using a magnetic particle concentrator (Dynal, Oslo, Norway) and cultured on collagen type I $\left(5 \mu \mathrm{g} / \mathrm{cm}^{2}\right)$ and human fibronectin (1 $\mu \mathrm{g} / \mathrm{cm}^{2}$ ) (Boehringer, Mannheim, Germany) coated glass chamber slides (Nunc, Naperville, Illinois), in the presence of EBM growth medium (Alessandri et al, 1998).

\section{Immunocytochemistry of CD34+/CD31- Cells}

Twenty-four hours and 7 to 10 days after plating on collagen-fibronectin substrata, the selected CD34+/ CD31 - cells were fixed in cold $4 \%$ paraformaldehyde in PBS, $\mathrm{pH} 7.4$, for 10 minutes at room temperature. They were then washed twice with PBS $(0.1 \%$ Triton-X, if necessary), blocked with 10\% normal goat serum (NGS) (Gibco, Grand Island, New York) and incubated with mouse anti-human CD31 and rabbit antiserum to human factor VIII (respectively, 1:100 and 1:80) (Sigma, St. Louis, Missouri) for 90 minutes at $37^{\circ} \mathrm{C}$. After two washings with PBS, the cells were incubated, respectively, with a 1:300 dilution of cyanine dye-labeled goat anti-mouse and anti-rabbit immunoglobulin G (IgG) (Cy2; Jackson Immunoresearch, West Grove, Pennsylvania) for 45 minutes at room temperature. Air-dried cells were then mounted with Fluorsave (Calbiochem, La Jolla, California) and photographed using a Zeiss Axiophot-2-microscope (Oberkochen, Germany). To exclude false positives produced by nonspecific binding of the secondary antibody, the same cells were stained in a similar manner with buffer substituting for primary antibody. Cells that were negative for the beads-selection (CD34-/CD31-) were used as negative controls.

\section{Flow Cytometry Analysis on Freshly Digested Aorta}

FACS was performed on the total mixed cell population obtained from collagenase-dispase digestion of an aortic fragment. Approximately $1 \times 10^{5}$ cells were collected, double-stained, and incubated in the dark for 30 minutes at $4^{\circ} \mathrm{C}$ with anti-CD34, FITC, and anti-CD31 PE (Becton Dickinson, San Jose, California) (dilution 1:10). The cells were then washed twice with PBS and analyzed for fluorescence by flow cytometry using a FACSscan (Becton Dickinson, Mountain View, California).

\section{Histology and Immunohistochemistry}

Formalin-fixed tissues were included in paraffin following standard histology techniques. Fourmicrometer serial sections were transferred to glass slides coated with poly-lysine and rehydrated by immersion in 100\% xylene and in a graded ethanol series (100\%, 95\%, 90\%, 80\%, and 70\%). Sections were then heat treated in a microwave cooker to enhance antigenicity and allow epitope unmasking: twice for 5 minutes each in $1 \mathrm{~mm}$ EDTA, pH 8, for CD31, CD34, and VWF antigens, and three times for 4 minutes each in $0.01 \mathrm{M}$ buffer citrate, $\mathrm{pH} 6$, for the Flk-1 antigen. 
Endogenous peroxidases were inhibited for 15 minutes at room temperature (RT) with 3\% hydrogen peroxide. Samples were then blocked for 20 minutes with $20 \%$ normal blocking serum, and the appropriate mixture of primary antibodies was subsequently added to serial sections: mouse anti-CD31 (clone JC/70A, DAKO), diluted 1:10, 30 minutes at RT; mouse anti-CD34 (Serotec, Raleigh, North Carolina), diluted $1: 50,30$ minutes at $37^{\circ} \mathrm{C}$; rabbit anti-vWF (DAKO), diluted 1:20, 30 minutes at RT; and mouse anti-Flk-1 (Santa Cruz Biotechnology, Santa Cruz, California), diluted 1:50, 45 minutes at RT. After washings, sections were incubated for 30 minutes with appropriate secondary antibodies conjugated to biotin and processed according to the avidin/biotin peroxidase complex method with kit reagents (mouse IgG and rabbit IgG Vectastain; Vector Laboratories, Burlingame, California). Peroxidase activity was shown with 3,3'-diaminobenzidine (Menarini-Biogenex, San Ramon, California) in PBS, and counterstaining was performed with hematoxylin-eosin. To exclude false positives produced by nonspecific binding of the secondary antibody, all of the tissues were treated in the same manner with buffer substituting for the primary antibody.

\section{Cord Formation on Matrigel}

Two hundred and seventy microliters of Matrigel (12.5 $\mathrm{mg} / \mathrm{ml}$ ) (Becton Dickinson, Bedford, Massachusetts) at $4^{\circ} \mathrm{C}$ were transferred to prechilled 24-well culture plates using sterile pipette tips that had been cooled to $-20^{\circ} \mathrm{C}$ before use. After gentle agitation to ensure even coating, plates were incubated for 30 minutes at $37^{\circ} \mathrm{C}$ to allow the Matrigel to solidify. CD34+/CD31cells were then seeded at a concentration of $6 \times$ $10^{4} /$ well in EBM growth medium (Alessandri et al, 1998). Cord formation was obtained after 24 hours of incubation.

\section{Electron Microscopy}

Selected cultured explants were fixed in $2.5 \%$ glutaraldehyde immediately after preparation, postfixed in osmium tetroxide, embedded in Epon-Aaraldite, and observed under a Zeiss CEM 902 electron microscope.

\section{Acknowledgements}

We thank Dr. C. Muneretto, Dr. L. Dei Cas, Dr. S. Bonardelli, and Dr. P.M. Giulini for human fetal blood vessel samples; Ms. E. Missana for technical assistance; and Mr. D. C. Ward for help with the English translation.

\section{References}

Alessandri G, Chirivi RGS, Castellani P, Nicolò G, Giavazzi R, and Zardi $L$ (1998). Isolation and characterization of human tumor-derived capillary endothelial cells: Role of oncofoetal fibronectin. Lab Invest 78:127-128.
Asahara T, Masuda H, Takahashi T, Kalka C, Pastore C, Silver M, Kearne M, Magner M, and Isner JM (1999). Bone marrow origin of endothelial progenitor cells responsible for postnatal vasculogenesis in physiological and pathological neovascularization. Circ Res 85:221-228

Asahara T, Murohara T, Sullivan A, Silver M, van der Zee R, Li T, Witzenbichler B, Schatteman G, and Isner JM (1997). Isolation of putative progenitor endothelial cells for angiogenesis. Science 275:964-967.

Balconi G, Spagnuolo R, and Dejana E (2000). Development of endothelial cell lines from embryonic stem cells: A tool for studying genetically manipulated endothelial cells in vitro. Arterioscler Thromb Vasc Biol 20:1443-1451.

Brown KJ, Maynes SF, Bezos A, Maguire DJ, Ford MD, and Parish $R$ (1996). A novel in vitro assay of human angiogenesis. Lab Invest 75:539-555.

Elsdale T and Bard J (1972). Collagen substrata for study of cell behavior. J Cell Biol 54:626-637.

Flamme I and Risau W (1992). Induction of vasculogenesis and hematopoiesis in vitro. Development 116:435-439.

Folkman J (1985). Toward an understanding of angiogenesis: Search and discovery. Perspect Biol Med 29:10-36.

Folkman J and Shing Y (1992). Angiogenesis. J Biol Chem 267:10931-10934.

Fuchs E and Segre A (2000). Stem cells: A new lease on life. Cell 100:143-155.

Gilbert SF (1997). Early vertebrate development: Mesoderm and endoderm. In: Developmental biology, 5th ed. Sunderland, MA: Sinauer Associates, 341-388.

Hirashima M, Kataoka H, Nishikawa S, Matsuyoshi N, and Nishikawa S (1999). Maturation of embryonic stem cells into endothelial cells in an in vitro model of vasculogenesis. Blood 93:253-263

LeDourain N (1973). A biological cell labelling technique and its use in experimental embryology. Dev Biol 30:217-222.

Mulligan RC (1993). The basic science of gene therapy. Science 260:926-931.

Nicosia RF, Tchao R, and Leighton J (1982). Histotypic angiogenesis in vitro: Light microscopy, ultrastructural, and radioautographic studies. In Vitro 18:538-549.

Nicosia RF and Ottinetti A (1990). Growth of microvessels in serum-free matrix culture of rat aorta: A quantitative assay of angiogenesis in vitro. Lab Invest 63:115-122.

Niklason LE (1999). Replacement arteries made to order. Science 286:1493-1494.

Nishikawa SI, Nishikawa S, Hirashima M, Matsuyoshi N, and Kodama $H$ (1998). Progressive lineage analysis by cell sorting and culture identifies FLK1+VE-cadherin + cells at a diverging point of endothelial and hemopoietic lineages. Development 125:1747-1757.

Pardanaud L, Altmann C, Kitos P, Dieterlen-Lièvre F, and Buck CA (1987). Vasculogenesis in the early quail blastodisc as studied with a monoclonal antibody recognizing endothelial cells. Development 100:339-349.

Peichev M, Naiyer AJ, Pereira D, Zhu Z, Lane WJ, Williams M, Oz MC, Hicklin DJ, Witte L, Moore MA, and Rafii S (2000). Expression of VEGFR-2 and AC133 by circulating human CD34(+) cells identifies a population of functional endothelial precursors. Blood 95:952-958. 
Risau W (1995). Differentiation of endothelium. FASEB J 9:926-933.

Risau W and Flamme I (1995). Vasculogenesis. Annu Rev Cell Dev Biol 11:73-91.

Weiss M and Orkin SH (1996). In vitro differentiation of murine embryonic stem cells. New approaches to old problems. J Clin Invest 97:591-595.
Yamashita J, Hiroshi I, Masanori H, Minetaro O, Satomi N, Takami Y, Makoto N, Kazuwa N, and Nishikawa SI (2000). Flk1-positive cells derived from embryonic stem cells serve as vascular progenitors. Nature 408:92-96. 